\title{
On the Validity of the Dynamical Mean-Field Approximation for Studying the Two-Dimensional Hubbard Model on a Square Lattice
}

\author{
A. N. Ribeiro and C. A. Macedo \\ Departamento de Fisica, Universidade Federal de Sergipe, 49100-000 Sao Cristovao-SE, Brazil \\ Correspondence should be addressed to A. N. Ribeiro, anevesr@gmail.com \\ Received 25 January 2010; Accepted 6 July 2010 \\ Academic Editor: Nigel E. Hussey
}

Copyright ( 2010 A. N. Ribeiro and C. A. Macedo. This is an open access article distributed under the Creative Commons Attribution License, which permits unrestricted use, distribution, and reproduction in any medium, provided the original work is properly cited.

The dynamical mean-field approximation (DMFA) becomes exact in the limit of infinite dimensions, and allows results to be obtained in a nonperturbative regime without the limitations normally found with exact diagonalization (ED) and quantum Monte Carlo (QMC) methods. In this paper, we investigate the applicability of the method to lattices with small coordination number in special situations. Specifically we use this approximation to study the two-dimensional (2D) Hubbard model on a square lattice far from half filling. In this situation, we calculate the specific heat and find that when the filling decreases, that is, antiferromagnetic correlations become less important, the agreement between DMFA and QMC results increases. Our results show that the DMFA can be a valuable technique for studying the thermodynamic properties of the Hubbard model also on a square lattice, but within a parameter range in which the antiferromagnetic correlations are not important.

\section{Introduction}

Strongly correlated electron systems (SCESs) present some of the most fascinating physical phenomena of condensedmatter physics. Some examples are itinerant ferromagnetism, Mott transition and superconductivity at high temperature $[1,2]$. The superconductivity at high temperature found in some cuprates has stimulated the research on the twodimensional (2D) Hubbard model on a square lattice because many researchers believe that this model is capable of capturing the essential physics needed in the description of the superconductivity of these materials. In SCES the energy of repulsion between electrons is comparable to or larger than the kinetic energy, and an approach which goes beyond theories that treat the electron-electron interaction as a perturbation is needed. Many approximations developed for studying SCES, such as arbitrary resummations of some class of diagrams, are not based on some extreme limit of the model in which the problem is simplified and can be solved in a controlled manner. Quite often it is not possible to confidently assess if a theoretical prediction reflects a true feature of the idealized Hamiltonian or if it is an artifact of the approximation used in its solution [2]. Exact numerical methods have been used intensively. Nevertheless, the exact diagonalization (ED) method for finite temperatures is limited to very small clusters and, far from half filling, the quantum Monte Carlo (QMC) method is restricted to rather high temperatures due to the minus-sign problem.

The dynamical mean-field approximation (DMFA) is a technique which allows results to be obtained in the nonperturbative regime without the limitations normally found in ED and QMC methods. The DMFA becomes exact in the limit of infinite lattice coordination (or infinite spatial dimensions), where the spatial fluctuations become frozen. In finite dimensions these fluctuations exist but are neglected by DMFA. Nevertheless, the on-site quantum fluctuations among the different atomic configurations are fully taken into account. The DMFA reduces the problem of the dynamics of interacting electrons on a lattice to a single-site problem with effective parameters self-consistently determined $[1,2]$.

The use of the DMFA for studying the 2D Hubbard model on a square lattice is questionable because, for this 
lattice, the coordination number is 4 , and numerical results have proven that important antiferromagnetic correlations occur at low temperatures. However, as the length of these correlations decreases when the mean number of electrons per site $n$ deviates from one (half-filled band) [3-5], we hope that at fillings where these correlations are not important the DMFA should be a good approximation for studying this system. This hypothesis has already been proposed by Pruschke et al. [6]. They compared results for the spectral function obtained by using DMFA and QMC and have found that the DMFA gives a rather accurate description of the dynamical properties of the 2D Hubbard model on a square lattice, as long as long-range spin correlations are not important. However, a study on the validity of this technique in calculations of static properties is still absent.

In this paper, we calculate the specific heat of the $2 \mathrm{D}$ Hubbard model on a square lattice using the DMFA in the paramagnetic phase for $n=0.75$ and 0.5 , with $U / t$ $=4,8$ and 12 . There are only a small number of studies of the specific heat of the 2D Hubbard model on a square lattice for a non-half-filled band. Duffy and Moreo calculated the specific heat by QMC [7], Mancini et al. utilized the composite operator method [8], and Bonca and Prelovsek used the finite-temperature Lanczos method with additional phase averaging for a system of $4 \times 4$ sites [9]. We find for $n=0.75$ results close to those obtained by Duffy and Moreo, and for $n=0.5$ the specific-heat curves calculated by DMFA are nearly the same as those calculated by QMC.

\section{Hubbard Model, DMFA, and Numerical Method}

The 2D Hubbard model on a square lattice is given by [10]

$$
H=\sum_{\mathbf{k}, \sigma} \varepsilon_{\mathbf{k}} c_{\mathbf{k}, \sigma}^{+} c_{\mathbf{k}, \sigma}+U \sum_{i} n_{i, \uparrow} n_{i, \downarrow},
$$

where $\varepsilon_{\mathbf{k}}=-2 t\left[\cos \left(k_{x} a\right)+\cos \left(k_{y} a\right)\right], t$ denotes the nearestneighbor hopping amplitude, $a$ denotes the lattice spacing, and the wave vectors of the first Brillouin zone $\mathbf{k}=\left(k_{x}, k_{y}\right)$ are obtained from the periodic contour condition; $c_{\mathbf{k}, \sigma}^{+}\left(c_{\mathbf{k}, \sigma}\right)$ is the creation (annihilation) operator of an electron in the Bloch state $(\mathbf{k}, \sigma) ; n_{i, \sigma}=c_{i, \sigma}^{+} c_{i, \sigma}$ is the number operator and determines the number of electrons at site $i$ with projection of spin $\sigma$ and $U$ designates the on-site electron-electron repulsion energy.

In the limit of infinite spatial dimensions, the self-energy of the Hubbard model $\sum_{\sigma}\left(\mathbf{k}, i \omega_{n}\right)$ becomes independent of the wave vector, $\sum_{\sigma}\left(\mathbf{k}, i \omega_{n}\right) \rightarrow \sum_{\sigma}\left(i \omega_{n}\right)[11,12]$. In the dynamical mean-field approximation, $\sum_{\sigma}\left(i \omega_{n}\right)$ is used as an approximation for the true self-energy of the Hubbard model on real lattices (lattices with finite dimensions). Physically, this approximation simplifies the spatial dependence of the correlations among electrons by neglecting spatial fluctuations (these fluctuations are only effectively frozen in the limit of infinite dimensions) but take full account of local quantum fluctuations. To compute $\sum_{\sigma}\left(i \omega_{n}\right)$, the Hubbard Hamiltonian is associated to single-site effective dynamics $[13,14]$. Using this simplified self-energy, the interacting single-site Green's function $G_{\sigma}\left(\mathbf{k}, i \omega_{n}\right)$ in the Hubbard model is given by

$$
G_{\sigma}\left(\mathbf{k}, i \omega_{n}\right)=\frac{1}{i \omega_{n}-\varepsilon_{\mathbf{k}}+\mu-\sum_{\sigma}\left(i \omega_{n}\right)},
$$

where $\mu$ denotes the chemical potential, $\omega_{n}=(2 n+1) \pi k_{B} T$ is the Matsubara frequency (here $n$ denotes an integer number), and $T$ denotes the absolute temperature. On the other hand, we regard $\sum_{\sigma}\left(i \omega_{n}\right)$ as the self-energy of a single-site problem with effective parameters. This problem can be described by the Anderson impurity Hamiltonian, which describes the dynamics of a magnetic impurity on site $i$ coupled to a conduction band [13]. The Anderson Hamiltonian is written as

$$
H_{A}=\sum_{l, \sigma} \tilde{\mathcal{\varepsilon}}_{l} c_{l, \sigma}^{+} c_{l, \sigma}+\sum_{l, \sigma} V_{l}\left(c_{l, \sigma}^{+} c_{i, \sigma}+c_{i, \sigma}^{+} c_{l, \sigma}\right)+U n_{i, \uparrow} n_{i, \downarrow}
$$

where, in the present context, $\widetilde{\varepsilon}_{l}$ and $V_{l}$ are effective parameters. These parameters are determined self-consistently, requiring that the impurity Green's function in the Anderson model, $G_{\sigma}\left(i \omega_{n}\right)_{\text {imp }}$, coincide with the on-site Green's function in the Hubbard model, $G_{i i, \sigma}\left(i \omega_{n}\right)=\Sigma_{k} G_{\sigma}\left(\mathbf{k}, i \omega_{n}\right)$. The Dyson's equation allows us to write

$$
G_{\sigma}\left(i \omega_{n}\right)_{\mathrm{imp}}=\frac{1}{\left[G_{\sigma}^{0}\left(i \omega_{n}\right)_{\mathrm{imp}}\right]^{-1}-\Sigma_{\sigma}\left(i \omega_{n}\right)_{\mathrm{imp}}},
$$

where $G_{\sigma}^{0}\left(i \omega_{n}\right)_{\mathrm{imp}}$ is the $U=0$ impurity Green's function and $\Sigma_{\sigma}\left(i \omega_{n}\right)_{\text {imp }}$ is the self-energy of the Anderson impurity model. The Green's function $G_{\sigma}^{0}\left(i \omega_{n}\right)_{\text {imp }}$ is given by

$$
G_{\sigma}^{0}\left(i \omega_{n}\right)_{\mathrm{imp}}=\left[i \omega_{n}+\mu-\sum_{l, \sigma} \frac{V_{l}^{2}}{i \omega_{n}-\widetilde{\varepsilon}_{l}}\right]^{-1}
$$

The equation $G_{i i, \sigma}\left(i \omega_{n}\right)=\sum_{\mathbf{k}} G_{\sigma}\left(\mathbf{k}, i \omega_{n}\right)$ together with equations (2), (4), and (5) and the Hamiltonian (3) form a closed system of equations for the on-site Green's function $G_{i i, \sigma}\left(i \omega_{n}\right)$ and the self-energy $\sum_{\mathbf{k}}\left(i \omega_{n}\right)$. These are the basic equations of the DMFA and the self-consistent solution is obtained as described below.

Using an initial set of parameters $\tilde{\mathcal{E}}_{l}, V_{l}$, the Green's functions $G_{\sigma}^{0}\left(i \omega_{n}\right)_{\text {imp }}$ and $G_{\sigma}\left(i \omega_{n}\right)_{\mathrm{imp}}$ are calculated, respectively, from (5) and by solving the Hamiltonian (3), for example using the ED method. Then, $\Sigma_{\sigma}\left(i \omega_{n}\right)_{\text {imp }}$ is calculated from (4) and, employing $\Sigma_{\sigma}\left(i \omega_{n}\right)=\Sigma_{\sigma}\left(i \omega_{n}\right)_{\text {imp }}$, the Green's function $G_{i i, \sigma}\left(i \omega_{n}\right)$ is calculated from (2). Making $G_{\sigma}\left(i \omega_{n}\right)_{\text {imp }}$ $=G_{i i, \sigma}\left(i \omega_{n}\right)$, a new Green's function $G_{\sigma}^{0}\left(i \omega_{n}\right)_{\text {imp }}$ is obtained from (4), and so new parameters $\left\{\widetilde{\varepsilon}_{l}, V_{l}\right\}$ are determined. The cycle is repeated until $G_{i i, \sigma}\left(i \omega_{n}\right)=G_{\sigma}\left(i \omega_{n}\right)_{\text {imp }}$ and $\Sigma_{\sigma}\left(i \omega_{n}\right)=$ $\Sigma_{\sigma}\left(i \omega_{n}\right)_{\text {imp. }}$. We solve this system of self-consistent equations by using an algorithm based on the exact numerical diagonalization of the Anderson Hamiltonian (3) with a finite number of sites presented by Caffarel and Krauth [15]. The program used by us is based on that indicated in [2]. 
We calculate the specific heat of the 2D Hubbard model on a square lattice with $200 \times 200$ sites in the paramagnetic phase for $n=0.75$ and 0.5 , with $U / t=4,8$, and 12. The internal energy $E$ was calculated numerically by a standard method using the retarded Green's function obtained by applying the analytic continuation $i \omega_{n} \rightarrow \omega+i \delta$ to (2) [16]. The specific heat $C(T)$ was first calculated by formula $C(T)=[E(T+\Delta T)-E(T)] /\left(N_{s} \Delta T\right)$, with $\Delta T=0.02$ $t / k_{B}\left(N_{s}\right.$ denotes the total number of sites), but this numerical differentiation did not produce a smooth curve for some parameters $(n, U)$. Thus, we calculate the specific heat by the method of Duffy and Moreo, that is, the calculated points of $E(T)$ are fitted to a polynomial of order 6 at low temperatures and to a polynomial of order 4 at high temperatures, and the specific heat is obtained by taking derivatives of these polynomials [7].

\section{Results and Discussions}

Figures 1 and 2 show the specific-heat curves calculated using DMFA and QMC for $n=0.75$ and 0.5 , respectively. The agreement between the results obtained by these two methods is good for $n=0.75$ and excellent for $n=0.5$. Since, at half filling $(n=1)$, the DMFA gives a qualitatively incorrect result because it predicts a Mott transition at $U_{c} \approx 12|t|$ [17]; we conclude that increases the quality of this approximation in the study of the 2D Hubbard model on a square lattice when the density $n$ decreases. This fact occurs because the DMFA neglects the antiferromagnetic correlations, and these correlations are more important at a filling close to one at low temperature.

At half filling, numerical results show that the 2D Hubbard model on a square lattice exhibits an antiferromagnetic phase at $T=0$. At a finite temperature, the Ghosh theorem asserts the destruction of this magnetic phase [18], although, long-range antiferromagnetic correlations survive at low temperature. When $n$ is reduced, the length of these correlations is also reduced [3-5]. Duffy and Moreo analyzed the specific heat obtained by QMC and conclude that at $U / t$ $=8$ important antiferromagnetic correlations are absent for $n \leq 0.75$ [7]. Therefore, since the DMFA neglects spatial fluctuations, it is not able to correctly describe the properties of the 2D Hubbard model on a square lattice for $n$ near to half filling at low temperatures, that is, within the parameter range where important antiferromagnetic correlations occur. However, for $n \leq 0.75$ our results show the validity of the DMFA for studying this system (at $U / t=12$ inclusive).

Figure 2 shows that, at quarter filling, the specific-heat curves possess only one peak. At this density, the curves obtained with DMFA and QMC present only a small difference in the height of this peak. However, White et al. showed by means of the spin-spin correlation function calculated by QMC that at $n=0.5$, with $U / t=4$ and $k_{B} T / t=0.1$, antiferromagnetic correlations are nearly absent [4]. Since, at this temperature, the specific heat value obtained by DMFA agrees with that obtained by QMC, and since the peak of the specific heat curve occurs at a higher temperature and the temperature effect is unfavorable to magnetic correlations,

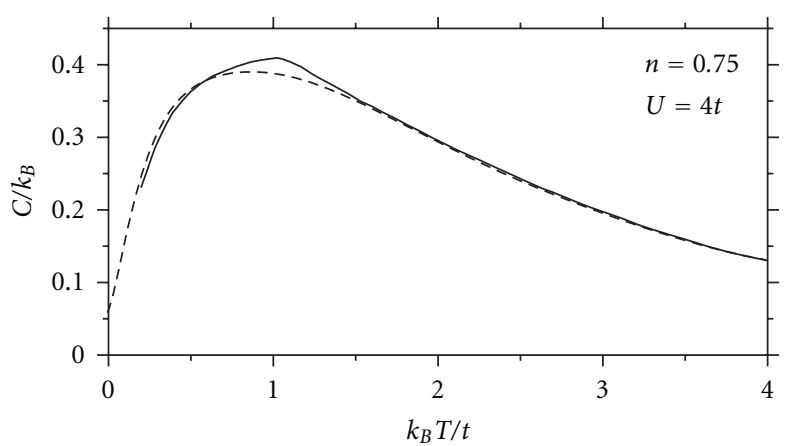

(a)

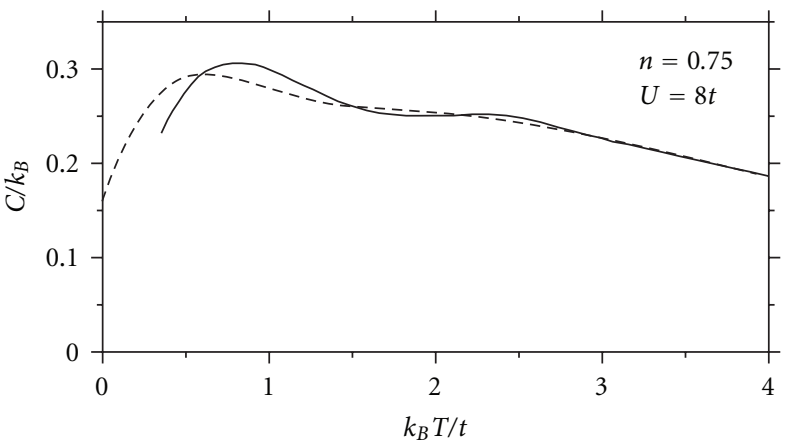

(b)

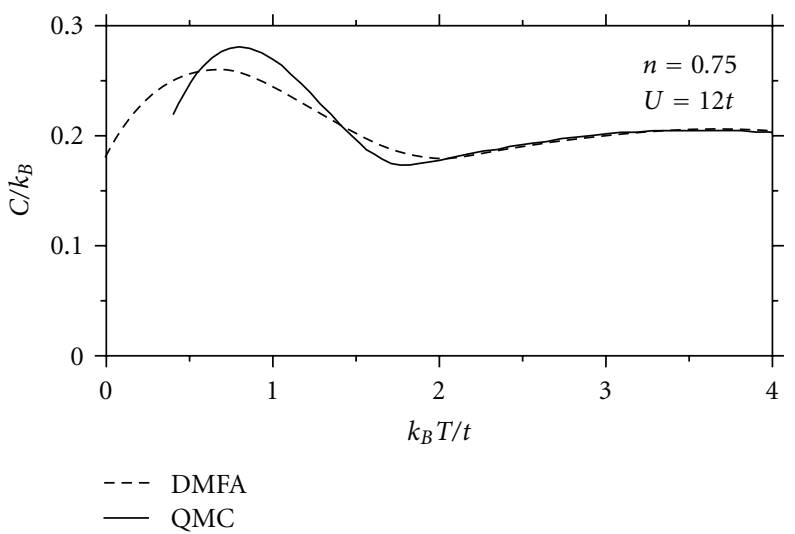

(c)

Figure 1: Specific heat $C$ versus temperature $T$ for $n=0.75$ with (a) $U / t=4$, (b) $U / t=8$, and (c) $U / t=12$. The full lines are QMC results taken from [7] and the dashed lines are our results calculated by DMFA.

we conclude that these correlations are not important in the formation of this peak, and consequently, this peak is mainly associated with local fluctuations (see Figure 2(a)). Since the DMFA takes full account of local quantum fluctuations, we believe that the difference between the specific heats obtained by DMFA and QMC at $n=0.5$ is mainly due to differences in the lattice sizes used in these calculations. The calculations with QMC and DMFA were carried out on a cluster of $6 \times 6$ sites and $200 \times 200$ sites, respectively [7]. At a density $n=0.75$, the agreement between the specific heat curves calculated by these two methods is excellent for $U / t=4$, and, for $U / t=8$ 


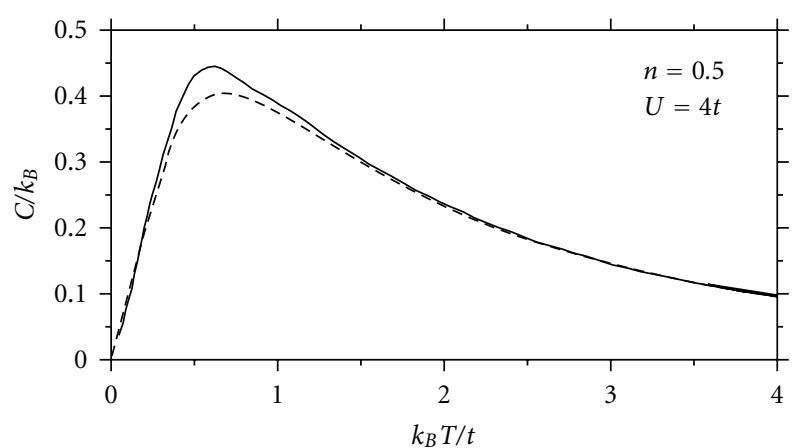

(a)

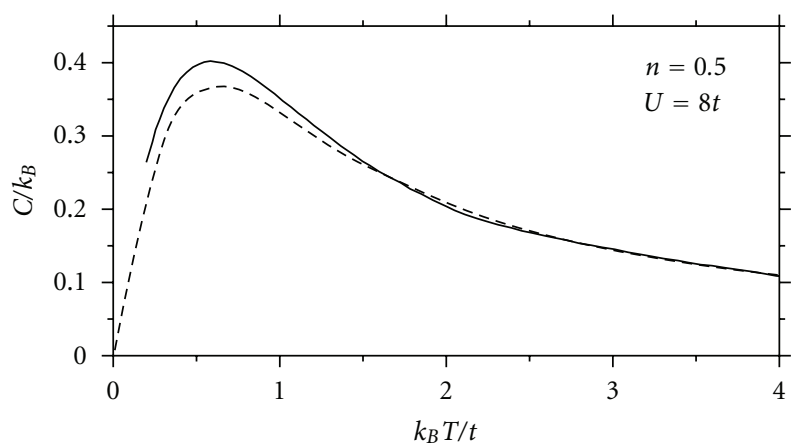

(b)

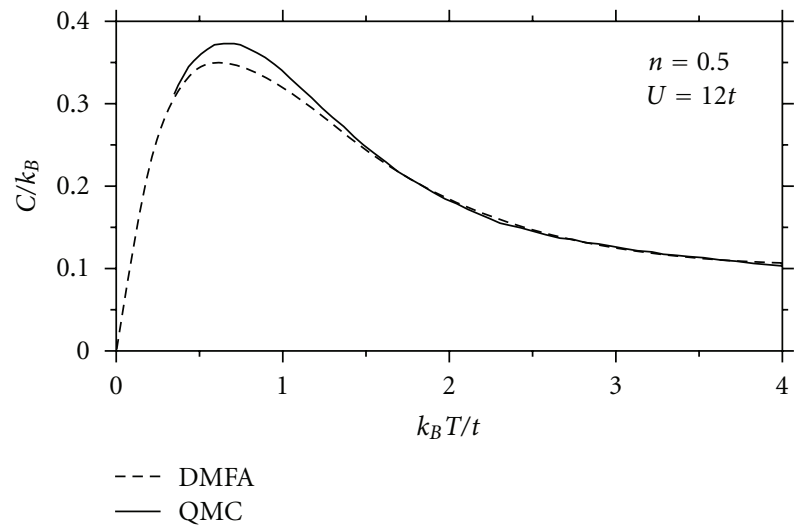

(c)

Figure 2: Specific heat $C$ versus temperature $T$ for $n=0.5$ with (a) $U / t=4$, (b) $U / t=8$, and (c) $U / t=12$. The full lines are QMC results taken from [7] and the dashed lines are our results calculated by DMFA.

and 12 the main difference is the position of the peak at low temperature (see Figure 1).

One believes that moving the filling away from $n=1$ not only the antiferromagnetic correlations are destroyed but also the Mott physics, which drives the system for the Mott insulating phase when $U / t$ is large. Nevertheless, the DMFA completely describes the local dynamics of the system, and as the Mott physics is associated with the intrasite (local) energy $U$, we think that the DMFA describes well the Mott Physics for any filling. However, studies which verify this hypothesis are important.

\section{Conclusions}

We calculate the dependence on temperature of the specific heat of the 2D Hubbard model on a square lattice far from half filling and compare it with QMC simulations. At $n=$ 0.75 , we find results very close to those obtained by using QMC, and at $n=0.5$ the specific heat curves calculated by using DMFA are nearly the same as those calculated by using QMC. Our results demonstrate that even in a twodimensional lattice, that is, with low coordination number, there is a parameter range where DMFA is capable of obtaining specific heat values as accurate as those obtained by QMC. This range is defined based on the nonexistence of important antiferromagnetic correlations. Since DMFA neglects spatial fluctuations, its use is valid only when these correlations are not important. Because DMFA permits the performance of calculations at low temperatures (without the limitations imposed by the minus-sign problem of the QMC method) and nearly in the thermodynamic limit, it should become a powerful method for studying the Hubbard model on a square lattice.

\section{Acknowledgment}

This paper was supported by the CAPES and CNPq (Brazilian agencies).

\section{References}

[1] Yu. A. Izyumov and E. Z. Kurmaev, "Materials with strong electron correlations," Physics-Uspekhi, vol. 51, no. 1, pp. 2356, 2008.

[2] A. Georges, G. Kotliar, W. Krauth, and M. J. Rozenberg, "Dynamical mean-field theory of strongly correlated fermion systems and the limit of infinite dimensions," Reviews of Modern Physics, vol. 68, no. 1, pp. 13-125, 1996.

[3] J. E. Hirsch, "Two-dimensional Hubbard model: numerical simulation study," Physical Review B, vol. 31, no. 7, pp. 44034419, 1985.

[4] S. R. White, D. J. Scalapino, R. L. Sugar, E. Y. Loh, J. E. Gubernatis, and R. T. Scalettar, "Numerical study of the twodimensional Hubbard model," Physical Review B, vol. 40, no. 1, pp. 506-516, 1989.

[5] A. Moreo, D. J. Scalapino, R. L. Sugar, S. R. White, and N. E. Bickers, "Numerical study of the two-dimensional Hubbard model for various band fillings," Physical Review B, vol. 41, no. 4, pp. 2313-2320, 1990.

[6] Th. Pruschke, Th. Obermeier, J. Keller, and M. Jarrell, "Spectral properties and bandstructure of correlated electron systems," Physica B, vol. 223-224, no. 1-4, pp. 611-615, 1996.

[7] D. Duffy and A. Moreo, "Specific heat of the two-dimensional Hubbard model," Physical Review B, vol. 55, no. 19, pp. 1291812924, 1997.

[8] F. Mancini, H. Matsumoto, and D. Villani, "Thermodynamics of the 2D Hubbard model," Journal of Physical Studies, vol. 3, no. 4, pp. 474-491, 1999.

[9] J. Bonča and P. Prelovšek, "Thermodynamics of the planar Hubbard model," Physical Review B, vol. 67, no. 8, Article ID 085103, 5 pages, 2003. 
[10] J. Hubbard, "Electron correlations in narrow energy bands," Proceedings of the Royal Society A, vol. 276, no. 1365, pp. 238257, 1963.

[11] W. Metzner and D. Vollhardt, "Correlated lattice fermions in $d=\infty$ dimensions," Physical Review Letters, vol. 62, no. 3, pp. 324-327, 1989.

[12] E. Müller-Hartmann, "Correlated fermions on a lattice in high dimensions," Zeitschrift für Physik B Condensed Matter, vol. 74, no. 4, pp. 507-512, 1989.

[13] A. Georges and G. Kotliar, "Hubbard model in infinite dimensions," Physical Review B, vol. 45, no. 12, pp. 6479-6483, 1992.

[14] M. Jarrell, "Hubbard model in infinite dimensions: a quantum Monte Carlo study," Physical Review Letters, vol. 69, no. 1, pp. 168-171, 1992.

[15] M. Caffarel and W. Krauth, "Exact diagonalization approach to correlated fermions in infinite dimensions: mott transition and superconductivity," Physical Review Letters, vol. 72, no. 10, pp. 1545-1548, 1994.

[16] G. D. Mahan, Many-Particle Physics, Plenum, New York, NY, USA, 1990.

[17] J. Merino, B. J. Powell, and R. H. McKenzie, "Ferromagnetism, paramagnetism, and a Curie-Weiss metal in an electrondoped Hubbard model on a triangular lattice," Physical Review $B$, vol. 73, no. 23, Article ID 235107, 22 pages, 2006.

[18] D. K. Ghosh, "Nonexistence of magnetic ordering in the one- and two-dimensional Hubbard model," Physical Review Letters, vol. 27, no. 23, pp. 1584-1587, 1971. 

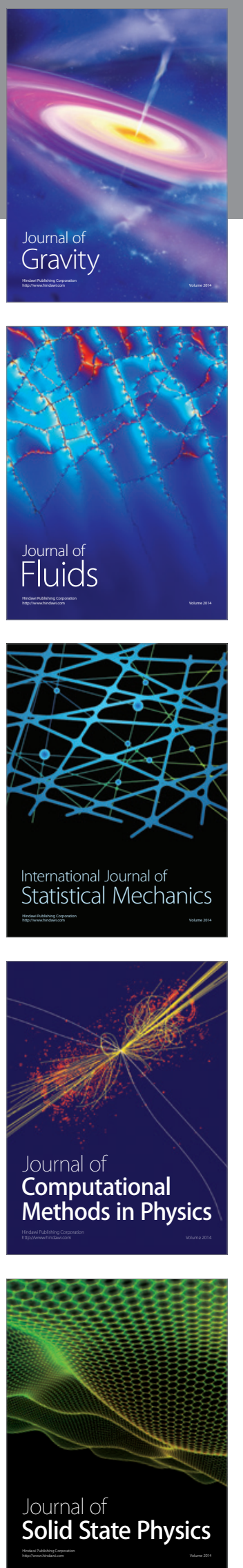

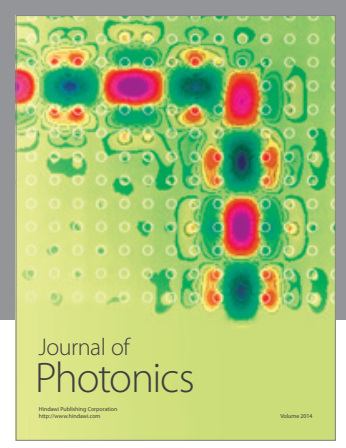

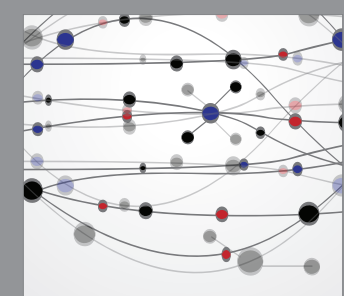

The Scientific World Journal
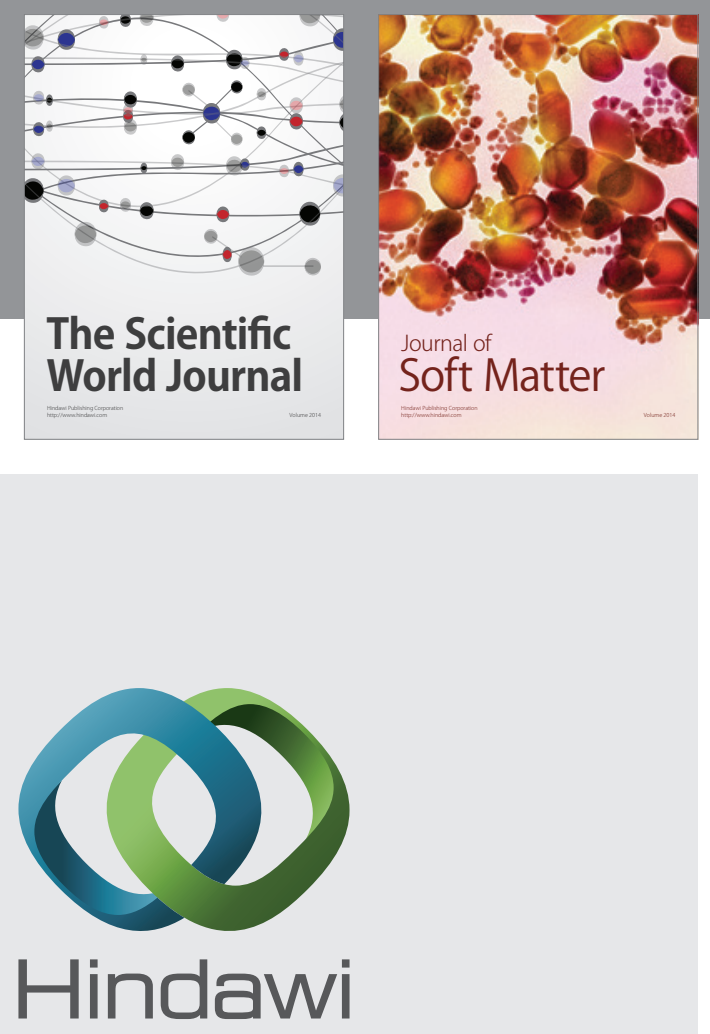

Submit your manuscripts at

http://www.hindawi.com
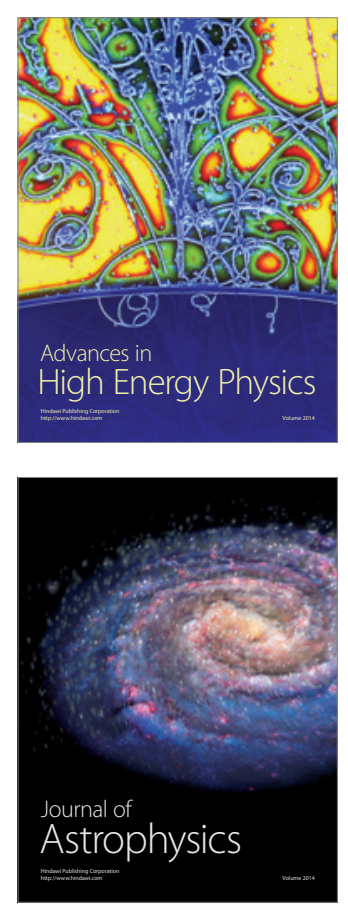
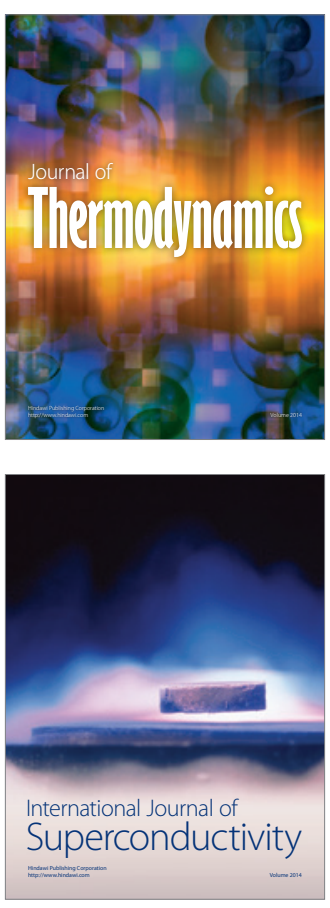
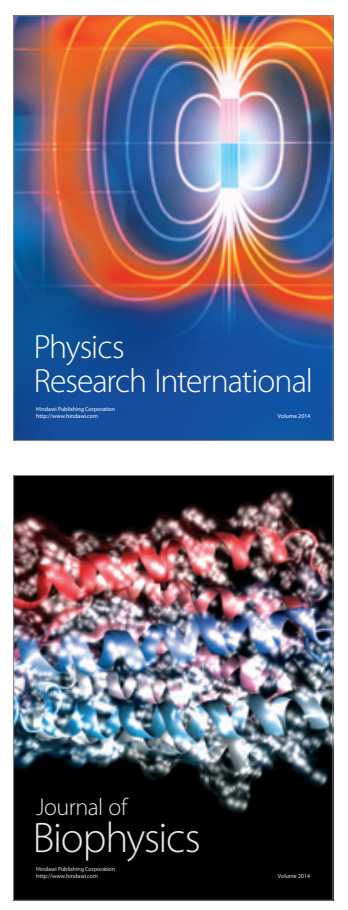
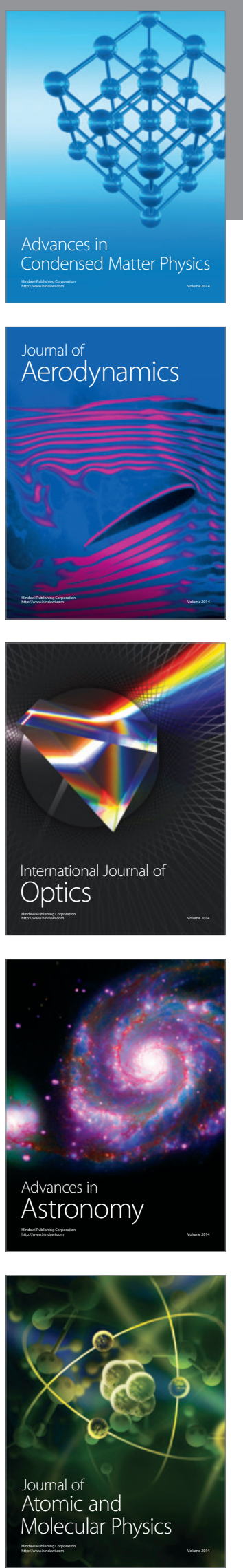